\title{
Tratamento de Carcinomatose Meníngea por Quimioterapia Intratecal Através de Catéter Reservatório de Ommaya: Experiência de um Centro Neurocirúrgico
}

\author{
Intratechal Chemotherapy Treatment Through an \\ Ommaya Reservoir Catheter for Meningeal \\ Carcinomatosis: A Single-Centre Experience
}

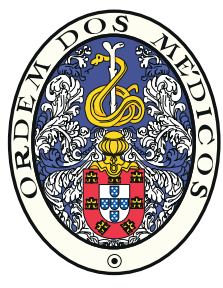

José Pedro LAVRADOR $₫ 1$,Nuno SIMAS ${ }^{1}$, Edson OLIVEIRA ${ }^{1}$, Manuel Herculano CARVALHO ${ }^{1}$

Acta Med Port 2016 Jul-Aug;29(7-8):456-460 - http://dx.doi.org/10.20344/amp.6733

\section{RESUMO}

Introdução: A carcinomatose meníngea consiste na infiltração de células tumorais ao longo das leptomeninges e espaço subaracnoideu, encontrando-se associada a uma sobrevida média de 2 - 5 meses. As indicações sobre a modalidade mais adequada de tratamento permanecem tema de discussão, sendo fundamental um correto conhecimento da história natural da doença e da dualidade risco-benefício para uma decisão terapêutica interdisciplinar.

Material e Métodos: Análise retrospectiva dos doentes com diagnóstico de carcinomatose meníngea com origem em tumores sólidos submetidos a colocação de catéter reservatório de Ommaya no período entre 2006 a 2014 no Serviço de Neurocirurgia do Hospital de Santa Maria.

Resultados: Foram operados 23 doentes com carcinomatose meníngea (19 mulheres, quatro homens) com idade média de 56,1 \pm 2,2 anos. A origem do tumor primário foi: mama - 16 doentes, pulmão - quatro doentes, estômago, bexiga e colo do útero - um doente cada. Não se verificaram complicações tais como infecção, hematomas intracranianos ou fístula de líquor. A sobrevida média dos 21 doentes falecidos à data foi de $26,4 \pm 7,7$ semanas (mínimo nove dias, máximo 118 semanas). A análise estatística não ajustada revelou que o sexo masculino esteve associado a pior prognóstico ( $p$ value $=0,0032$ ), enquanto a análise ajustada mostrou que a origem na mama esteve associada a melhor prognóstico $(p$ value $=0,036)$ quando comparada com as outras etiologias (HR: 4,36 $\pm 3,06 ; 95 \%$ IC: 1,10 - 17,25). Um maior tempo de evolução de doença primária até ao diagnóstico esteve associado a melhor prognóstico, apesar de não ter atingido significado estatístico.

Discussão: Apesar da colocação de catéter reservatório de Ommaya ser um procedimento com baixo risco de complicações, a resposta à quimioterapia intratecal é limitada e o prognóstico da doença poderá não justificar um procedimento cirúrgico num doente com mau estado funcional. A relação da sobrevida global com a origem do tumor primário sugere que o prognóstico da carcinomatose meníngea deve ser considerado no contexto da doença sistémica e não como uma doença isolada. O pior prognóstico do sexo masculino pode justificar-se pela menor incidência do tumor da mama neste género.

Conclusão: A carcinomatose meníngea está associada a um mau prognóstico. Origem primária na mama, maior tempo de evolução de doença primária e idade $<60$ anos estiveram associados a sobrevida mais longa.

Palavras-chave: Carcinomatose Meníngea/quimioterapia; Injecções Espinhais; Metástase Neoplásica/quimioterapia; Resultado do Tratamento.

\section{ABSTRACT}

Introduction: Meningeal carcinomatosis is defined as tumour cells infiltration of leptomeninges and subarachnoid space. It is normally related with poor survival (2 - 5 months). The best multidisciplinary treatment for this condition is a matter of discussion. Patient's condition and the natural history of the disease should be considered in the decision making process.

Material and Methods: Retrospective cohort analysis of patients submitted to Ommaya Reservoir placement due to systemic solid tumour meningeal carcinomatosis between 2006 and 2014.

Results: Twenty three patients were included (19 females, four males) with median age of $56.1 \pm 2.2$ years. The primary tumour was: breast - 16 patients, lung - four patients, stomach, bladder and cervix - one patient each. No complications were seen (infection, intracranial haematoma or CSF fistula). The median survival was $26.4 \pm 7.7$ weeks, range between nine days and 118 weeks $(21 / 23$ patients). Male gender was related to poor prognosis in crude analysis ( $p$ value $=0.0032$ ). Breast adenocarcinoma was related with better prognosis in adjust analysis ( $p$ value $=0.036, \mathrm{HR}: 4.36 \pm 3.06$; $95 \%$ IC: $1.10-17.25$ ). Longer time between initial tumour and meningeal carcinomatosis diagnosis was related to a better outcome but without statistical significance.

Discussion: Despite the low complication rate of Ommaya reservoir placement, the poor response to chemotherapy and the disease prognosis should be considered in patients with poor functional status. The relationship observed between the primary tumour and the overall survival supports that meningeal carcinomatosis should not be considered a disease by itself but always in the context of a systemic disease. The low incidence of breast cancer in male population might be related with it poorer prognosis.

Conclusion: Meningeal carcinomatosis has a poor prognosis. Breast adenocarcinoma, longer time between initial tumour and meningeal carcinomatosis diagnosis, and age $<60$ years were related with longer survival.

Keywords: Injections, Spinal; Meningeal Carcinomatosis/drug therapy; Neoplasm Metastasis/drug therapy; Treatment Outcome.

\footnotetext{
1. Serviço de Neurocirurgia. Hospital de Santa Maria. Centro Hospitalar Lisboa Norte. Lisboa. Portugal.

$\triangle$ Autor correspondente: José Pedro Lavrador. jose.pedro.lavrador@gmail.com

Recebido: 20 de junho de 2015 - Aceite: 15 de novembro de 2015 | Copyright @ Ordem dos Médicos 2016
} 


\section{INTRODUÇÃO}

A carcinomatose meníngea (CM) consiste na infiltração do espaço subaracnoideu e das leptomeninges por células provenientes da metastização de tumores sistémicos. ${ }^{1}$ É uma forma de metastização pouco frequente, ocorrendo em $5 \%-10 \%$ dos doentes com tumores sistémicos. ${ }^{2,3}$ No entanto, verificou-se um aumento recente do seu diagnóstico atribuído à melhoria do tratamento da doença sistémica, da suspeição clínica e acuidade diagnóstica. ${ }^{4} \mathrm{~A}$ CM encontra-se associada à progressão tumor primitivo em $75 \%$ dos casos e a outras manifestações neuro-oncológicas: lesões focais no sistema nervoso central (SNC), alterações da dinâmica do líquor em 33\% dos casos e encefalopatia em $15 \%$ dos casos. ${ }^{2}$ A capacidade de metastização para o espaço subaracnoideu não é semelhante em todos os tumores. No que respeita à origem do tumor primário, $50 \%$ dos casos provêm da mama e $27 \%$ - $30 \%$ do pulmão, apesar de ser o melanoma o tumor com maior tendência para disseminação leptomeníngea. ${ }^{5}$ No entanto, estes resultados podem traduzir diferenças na história natural da doença e na capacidade de diagnóstico. O prognóstico desta complicação é reservado, com sobrevidas médias reportadas de $6-7$ semanas, ${ }^{6}$ podendo estender-se até aos $3,3-4,5$ meses em subgrupos mais favoráveis. ${ }^{7,8}$

O melhor tratamento para esta forma de metastização não é consensual. O prognóstico inerente à $\mathrm{CM}$ e as complicações neurológicas associadas podem fazer do tratamento paliativo a melhor abordagem em muitas situações. Alguns autores apontam a evidência imagiológica de $\mathrm{CM}$ e um baixo performance status como fatores que favorecem uma abordagem conservadora. ${ }^{6}$ No entanto, é também importante identificar o subgrupo de doentes que poderá beneficiar em termos de sobrevida e qualidade de vida com um tratamento dirigido. Neste subgrupo são considerados os doentes com: bom performance status, idade $<60$ anos, doença sistémica mínima com opções razoáveis de tratamento, ausência de défices neurológicos major, tumor da mama, tempo de evolução da doença primária > 1 ano, ausência de hidrocefalia, captação de contraste linear (versus nodular), ausência de imagem sugestiva de CM em ressonância magnética, proteinorraquia pouco elevada e quimioterapia intratecal. $., 6,22$

Não existe consenso sobre a melhor via de administração de quimioterapia para o tratamento da carcinomatose meníngea. A literatura que compara a administração endovenosa em alta dose com a administração intratecal não é conclusiva, sendo reportados resultados antagónicos. ${ }^{6,9,10}$ Apesar de não existir uniformidade relativamente ao risco aumentado de toxicidade com a administração sistémica de quimioterapia em alta dose, o seu risco real - atingindo os $36,3 \%$ no estudo de Boogerd $W$ et $a^{9}$ - fez ressurgir o interesse pela administração intratecal de quimioterapia. ${ }^{11}$ Três fármacos têm sido usados de forma sistemática com administração intratecal no tratamento da doença metastática leptomeníngea - metotrexato, citarabina e thiotepa - e outros agentes têm sido testados, nomeadamente diferentes tipos de anticorpos monoclonais (rituximab e trastuzu- mab). ${ }^{12,13}$ A quimioterapia intratecal pode ser administrada através da realização de punções lombares seriadas ou por punção de sistemas colocados no interior de espaços de líquor com reservatórios subcutâneos, nomeadamente, cateteres reservatórios de Ommaya ou similares. Estes têm como principais vantagens o conforto do doente (evitando punções lombares repetidas), a segurança em cada administração, menor variabilidade dos parâmetros farmacocinéticos e melhoria da distribuição dos fármacos nos espaços de líquor na ausência de alterações da circulação do líquor. ${ }^{14}$ As principais desvantagens são o risco infeccioso e de disfunção, situado nos $10 \% .^{12}$

Tratando-se de uma progressão de uma doença maligna primária, a CM não é curável com tratamento dirigido. Ainda assim, é possível obter melhoria imagiológica e negativização da citologia do LCR após tratamento em alguns doentes. Além disso, a quimioterapia intratecal e a radioterapia focal podem melhorar a qualidade de vida e aumentar a sobrevida. ${ }^{11}$

O objetivo principal do estudo é analisar a sobrevida dos doentes com diagnóstico de carcinomatose meníngea submetidos a colocação de reservatório de Ommaya para realização de quimioterapia Intratecal. Como objetivos secundários destaca-se a identificação de fatores preditivos de resposta à terapêutica Intratecal e subsequente definição de subgrupos de melhor prognóstico.

\section{MATERIAL E MÉTODOS}

Estudo de coorte retrospetivo entre janeiro de 2005 e outubro de 2014 de doentes tratados no Serviço de Neurocirurgia, Hospital de Santa Maria, Centro Hospitalar Lisboa Norte. A informação clínica foi obtida a partir dos registos médicos efetuados em internamento e ambulatório dos doentes referenciados e submetidos a intervenção cirúrgica neste serviço. Foram considerados para colocação de cateter reservatório de Ommaya doentes com bom estado geral (índice de Karnofsky > 70), sem defeito neurológico major, com doença sistémica controlada e sem contra-indicação sistémica para realização de cirurgia. Os critérios de inclusão foram: colocação de cateter reservatório de Ommaya, diagnóstico de CM por citologia do líquor ou por imagiologia, diagnóstico prévio de tumor primário sólido. O critério de exclusão foi a origem hematológica do tumor primário. Foram avaliadas as seguintes variáveis: sexo, idade, tumor primitivo, sinais e sintomas clínicos no momento do diagnóstico, presença de células neoplásicas no líquor, tempo até ao diagnóstico de $\mathrm{CM}$ e sobrevida. $\mathrm{O}$ agente anti-neoplásico utilizado em todos os doentes foi o metotrexato intratecal na dose de $12 \mathrm{mg} / \mathrm{m}^{2}$.

Foi feita uma análise estatística descritiva das principais variáveis, sendo reportadas as respetivas médias e desvios-padrão e/ou proporções. A comparação das diferenças entre as médias do tempo para o diagnóstico de $\mathrm{CM}$ foi feita com utilizando o $t$ test. A análise de sobrevida foi feita através de curvas de Kaplan-Meyer, utilizando-se o LogRank Test para a análise comparativa entre grupos. 


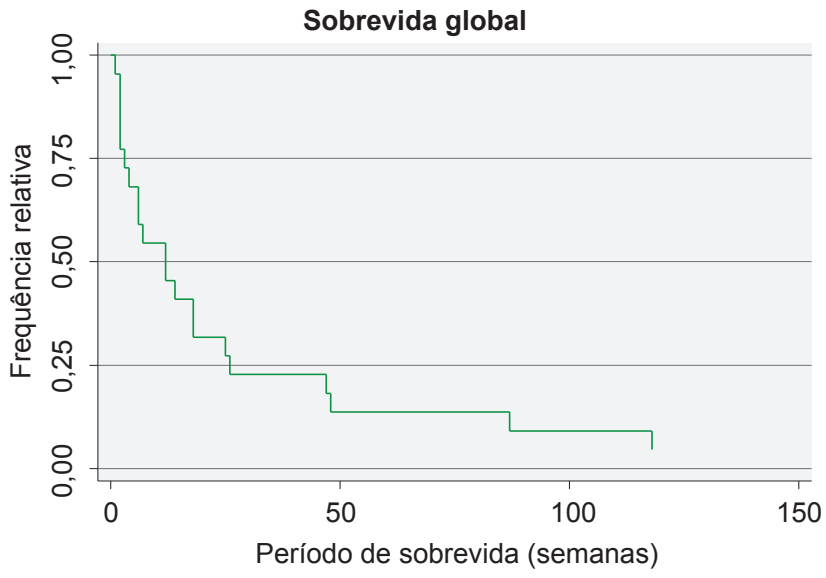

Figura 1 - Sobrevida global dos doentes submetidos a tratamento com quimioterapia intratecal através de cateter reservatório de Ommaya

A análise multivariável foi feita utilizando o Cox Proportional Hazards Ratio. Foram realizados dois ajustamentos distintos: o primeiro teve em consideração as variáveis 'idade' e 'tempo para o diagnóstico' como variáveis contínuas e o segundo considerou-as como variáveis dicotómicas (Idade $<65$ ou $\geq 65$ anos; tempo para o diagnóstico $<5$ ou $\geq 5$ anos). Para a realização desta análise foi utilizado o software estatístico STATA 13.0.

\section{RESULTADOS}

No período considerado, foram colocados catéteres reservatórios de Ommaya (CRO) em 51 doentes, em 23 dos quais por carcinomatose meníngea de tumores sólidos sistémicos, que constituiu o grupo de estudo. Esta amostra é constituída por 19 mulheres e quatro homens, com idade média de $56,1 \pm 2,2$ anos $(55,5 \pm 2,6$ nas mulheres e 59 $\pm 1,9$ nos homens). A citologia do LCR foi positiva em 19 dos 23 doentes. A mama foi a localização mais frequente do tumor primário (16/23), seguido pelo pulmão (4/23). A sintomatologia relacionada com hipertensão intracraniana (cefaleias e vómitos) e a disfunção dos nervos cranianos $\mathrm{IX}, \mathrm{X}$ e $\mathrm{XI}$ foram as formas de apresentação mais frequentes (9/23). O tempo médio para o diagnóstico de CM após o diagnóstico inicial da doença primária foi 4,5 \pm 0,9 anos, sendo maior nos doentes com neoplasia primária da mama comparativamente com os restantes $(5,2 \pm 1,2$ anos versus 2,4 \pm 1,2 anos) embora esta diferença não fosse estatisticamente significativa $(p=0,1120)$. A sobrevida média foi de 26,4 \pm 7,7 semanas (mínimo nove dias, máximo 118 semanas) (Fig. 1). Três dos quatro doentes com CM por adenocarcinoma do pulmão apresentaram sobrevida inferior a duas semanas. Nas duas doentes com CM por neoplasia da mama e idade superior a 70 anos, a sobrevida foi inferior a quatro semanas. Não se verificaram complicações tais como infecção, hematomas intracranianos ou fístula de líquor (Tabela 1).

A análise de sobrevida não ajustada não revelou diferenças estatisticamente significativas entre os diferentes grupos quando consideradas as variáveis tumor primitivo,
Tabela 1 - Características da Amostra

\begin{tabular}{|c|c|}
\hline \multicolumn{2}{|l|}{ Características da amostra } \\
\hline \multicolumn{2}{|l|}{ Sexo } \\
\hline Masculino & 4 \\
\hline Feminino & 19 \\
\hline Idade (anos) & $56,1 \pm 2,2$ \\
\hline Masculino & $59,0 \pm 1,9$ \\
\hline Feminino & $55,5 \pm 2,6$ \\
\hline \multicolumn{2}{|l|}{ Tumor primitivo (localização) } \\
\hline Mama & 16 \\
\hline Pulmão & 4 \\
\hline \multicolumn{2}{|l|}{ Outros: } \\
\hline Estômago & 1 \\
\hline Colo do útero & 1 \\
\hline Bexiga & 1 \\
\hline \multicolumn{2}{|l|}{ Sinais e sintomas à apresentação } \\
\hline Nervos cranianos & 9 \\
\hline Cefaleias e vómitos & 9 \\
\hline Desorientação/confusão & 2 \\
\hline Meningismo & 1 \\
\hline $\begin{array}{l}\text { Hipostesia dos membros inferiores e } \\
\text { paraparésia }\end{array}$ & 1 \\
\hline \multicolumn{2}{|l|}{ Células neoplásicas no líquor* } \\
\hline Presente & 12 \\
\hline Ausente & 3 \\
\hline Tempo até ao diagnóstico (anos) & $4,5 \pm 0,9$ \\
\hline Mama & $5,2 \pm 1,2$ \\
\hline Outros & $2,4 \pm 1,2$ \\
\hline Sobrevida (semanas) & $26,4 \pm 7,7$ \\
\hline Mama & $29,7 \pm 8,6$ \\
\hline Outros & $19,1 \pm 16,5$ \\
\hline
\end{tabular}

Oito doentes sem informação clínica

Tabela 2 - Análise de sobrevida não ajustada

\begin{tabular}{ll}
\hline Análise de sobrevida não ajustada & \\
\hline Tumor primitivo & $p=0,0689$ \\
Idade* $^{*}$ & $p=0,8389$ \\
Género & $\boldsymbol{p}=\mathbf{0 , 0 0 3 2}$ \\
Células neoplásicas no líquor & $p=0,5206$ \\
Tempo para diagnóstico** & $p=0,2884$ \\
\hline
\end{tabular}

$<65$ anos e $\geq 65$ anos

$<5$ anos $e \geq 5$ anos

idade, presença de células neoplásicas no líquor e tempo para o diagnóstico $(p>0,05)$. No que respeita o género, foi considerada uma diferença estatisticamente significativa ( $p$ $=0,0032$ ), com os homens a terem sobrevidas mais reduzidas (Tabela 2).

No que respeita ao ajustamento estatístico considerando as variáveis contínuas, nenhuma das variáveis consideradas atingiu uma diferença estatisticamente significativa $(p>0,05)$, apesar da tendência verificada para um melhor 
Tabela 3 - Análise de sobrevida ajustada (modelo final com ajustamento para tumor primitivo, idade, tempo entre o diagnóstico primário de doença oncológica e diagnóstico de CM e presença de células neoplásicas no LCR)

\begin{tabular}{|c|c|c|c|c|}
\hline \multicolumn{5}{|l|}{ Análise de sobrevida ajustada } \\
\hline & HR & Erro padrão & $p>|z|$ & {$[95 \%$ IC] } \\
\hline Tumor primitivo & 4,49 & 3,70 & 0,069 & $0,89-22,63$ \\
\hline Idade & 1,02 & 0,06 & 0,702 & $0,92-1,14$ \\
\hline Tempo para diagnóstico & 0,97 & 0,10 & 0,800 & $0,79-1,20$ \\
\hline Células neoplásicas no líquor & 3,92 & 4,85 & 0,269 & $0,35-44,21$ \\
\hline
\end{tabular}

Tabela 4 - Análise de sobrevida ajustada por categorias (modelo final com ajustamento para tumor primitivo, idade, tempo entre o diagnóstico primário de doença oncológica e diagnóstico de CM e presença de células neoplásicas no LCR)

\begin{tabular}{|c|c|c|c|c|}
\hline \multicolumn{5}{|c|}{ Análise de sobrevida ajustada por categorias } \\
\hline & $\mathrm{HR}$ & Erro padrão & $p>|z|$ & {$[95 \% \mathrm{IC}]$} \\
\hline Tumor primitivo & 4,36 & 3,06 & 0,036 & $1,10-17,25$ \\
\hline Idade* & 1,54 & 1,81 & 0,710 & $0,16-15,33$ \\
\hline Tempo para diagnóstico** & 0,95 & 0,76 & 0,953 & $0,20-4,55$ \\
\hline Células neoplásicas no líquor & 2,70 & 3,06 & 0,380 & $0,29-24,87$ \\
\hline
\end{tabular}

${ }^{*}<65$ anos e $\geq 65$ anos

** $<5$ anos $e \geq 5$ anos

prognóstico em termos de sobrevida dos doentes com tumor primário da mama ( $p=0,069)$ (Tabela 3 ). No ajustamento considerando categorias dicotómicas nas variáveis idade e tempo para o diagnóstico, os doentes com tumor primário da mama apresentaram um melhor prognóstico ( $p$ $=0,036$ ), tendo os doentes com tumores doutros órgãos sólidos um risco de mortalidade aumentado (HR: 4,36 \pm 3,06; 95\% IC: 1,10 - 17,25) (Fig. 2, Tabela 4).

\section{DISCUSSÃO}

A CM é uma forma de metastização de um tumor sistémico e não uma doença em si própria. Para a realização de uma abordagem terapêutica adequada às necessidades de cada doente, urge definir critérios que permitam uma selecção dos doentes que possam beneficiar de estratégias de tratamento mais agressivas. Durante o período de tempo considerado, a principal indicação para a colocação de CRO foram os tumores hematológicos (28/51). A indicação para a sua utilização nas doenças hematológicas

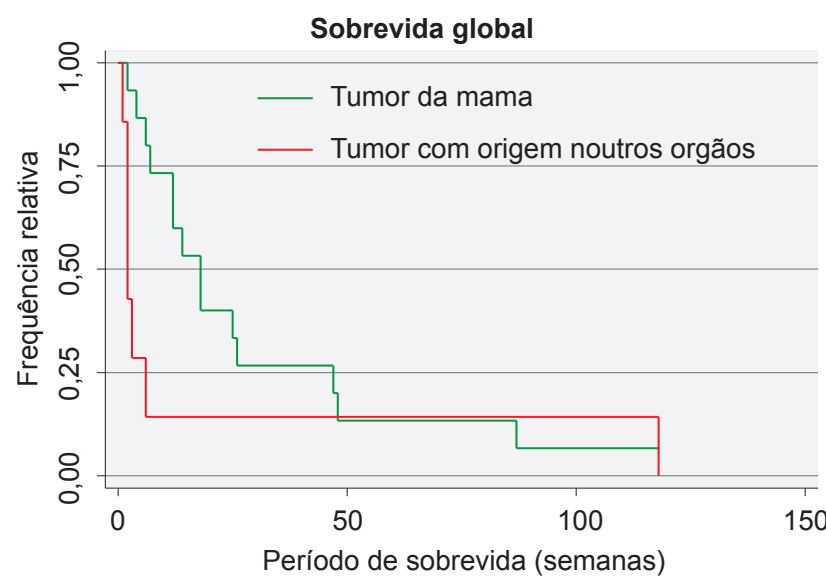

Figura 2 - Sobrevida global dos doentes submetidos a tratamento com quimioterapia intratecal através de cateter reservatório de Ommaya tendo em conta o tipo de tumor oncológicas disseminadas no sistema nervoso central está bem documentada na literatura, aumentando a sobrevida e a probabilidade de cura - sobrevida de $90 \%$, nas crianças, e de $50 \%$ nos adultos na leucemia linfoblástica aguda aos cinco anos. ${ }^{16}$ Este quadro é diferente do já exposto para a CM que se assume como um evento tardio e muitas vezes terminal na história natural dos tumores sólidos. ${ }^{6}$

A sobrevida média da população estudada $(26,4 \pm 7,7$ semanas) foi superior às referidas na literatura (6 - 7 semanas até 3,3 - 4,5 meses). ${ }^{6,7,8}$ Este facto pode ser explicado por um viés de selecção no sentido dos doentes com melhor prognóstico e por uma melhoria no tratamento da doença oncológica. As características demográficas reflectem esse viés de selecção, mostrando um predomínio do tumor da mama e uma distribuição demográfica com predomínio do sexo feminino na idade adulta $(55,5 \pm 2,6$ anos). Esta distribuição é semelhante à reportada na literatura, tendo em conta o melhor prognóstico da neoplasia da mama em termos de sobrevida global e progressão da doença de base ${ }^{17}$ e o fato de alguns autores referirem a idade $>60$ anos como um fator de mau prognóstico. ${ }^{18} \mathrm{~A}$ análise estatística não ajustada mostrou que os homens têm sobrevidas estatisticamente significativas mais curtas. No entanto, após a realização de ajustamento para os fatores de confundimento identificados, apenas a origem do tumor primitivo mostrou significância estatística, estando a mama associado a melhor prognóstico. Este achado é suportado por outras séries da literatura ${ }^{5,7,8,19}$ e poderá justificar as diferenças encontradas na análise não ajustada no que se refere ao género.

O tempo médio para o diagnóstico de $\mathrm{CM}$ foi diferente entre os tumores com origem na mama e os restantes apesar de não ter atingido significância estatística entre eles (5,2 $\pm 1,2$ vs 2,4 $\pm 1,2$ anos), provavelmente pelo número limitado de doentes incluídos. Na literatura são encontrados valores que oscilam entre os 7,4 anos para tumores de 
mama ${ }^{19}$ e 17 meses para tumores do estômago. ${ }^{20} \mathrm{O}$ intervalo livre de doença encontra-se inversamente relacionado com a agressividade do tumor primitivo, condicionando o prognóstico vital ${ }^{21}$ e apoiando o carcinoma da mama como fator de melhor prognóstico.

As complicações peri-operatórias, infecções ou disfunções associadas ao CRO são a principal preocupação associada a este procedimento. No entanto, no grupo de estudo não ocorreu nenhuma complicação ao contrário de outras séries da literatura, ${ }^{12}$ sustentando a segurança do procedimento.

Como principais limitações inerentes ao estudo, salientamos o número limitado de doentes e a utilização de um agente anti-neoplásico homogéneo no tratamento de $\mathrm{CM}$ de diferentes etiologias. No entanto, o metotrexato é o agente mais utilizado e referido na literatura para o tratamento da CM. Salientamos que este é um dos primeiros estudos sobre esta temática realizados na população portuguesa, permitindo comparar a nossa prática e os nossos resultados com os reportados na literatura.

Este estudo sustenta a instituição de um tratamento intensivo da CM nos doentes com melhor prognóstico - neoplasia da mama, tempo de evolução da doença primária $>1$ ano e idade < 60 anos - melhorando os sintomas e proporcionando uma aumento da sobrevida.

\section{CONCLUSÃO}

A CM tem uma resposta terapêutica limitada e está associada a um mau prognóstico. É fundamental a selecção de grupos de doentes que possam beneficiar de um tratamento mais intensivo, nomeadamente, através da quimioterapia intratecal através de CRO. No presente estudo, a origem primária na mama, o maior tempo de evolução de doença primária e a idade $<60$ anos estiveram associados a um benefício em termos de sobrevida.

\section{AGRADECIMENTOS}

Os autores gostariam de agradecer a José Miguéns pelo seu apoio na revisão e preparação do manuscrito.

\section{PROTECÇÃO DE PESSOAS E ANIMAIS}

Os autores declaram que os procedimentos seguidos estavam de acordo com os regulamentos estabelecidos pelos responsáveis da Comissão de Investigação Clínica e Ética e de acordo com a Declaração de Helsínquia da Associação Médica Mundial.

\section{CONFIDENCIALIDADE DOS DADOS}

Os autores declaram ter seguido os protocolos do seu centro de trabalho acerca da publicação de dados.

\section{CONFLITOS DE INTERESSE}

Os autores declaram não terem qualquer conflito de interesse relativamente ao presente artigo.

\section{FONTES DE FINANCIAMENTO}

Os autores declaram não ter recebido subsídios ou bolsas para a elaboração do artigo.

\section{REFERÊNCIAS}

1. Shen TY, Young YH. Meningeal carcinomatosis manifested as bilateral progressive sensorineural hearing loss. Am J Otol. 2000;21:510-2.

2. Chamberlain MC. Neoplastic meningitis. Oncologist. 2008;13:967-77.

3. Gleissner B, Chamberlain MC. Neoplastic meningitis. Lancet Neurol. 2006;5:443-52.

4. Glantz MJ, Van Horn A, Fisher R, Chamberlain MC. Route of intracerebrospinal fluid chemotherapy administration and efficacy of therapy in neoplastic meningitis. Cancer. 2010;116:1947-52.

5. Silvani A, Caroli M, Gaviani P, Fetoni V, Merli R, Riva M, et al. Neoplastic meningitis from solid tumors: a prospective clinical study in lombardia and a literature review on therapeutic approaches. J Drug Deliv. 2013;2013:147325

6. Waki F, Ando M, Takashima A, Yonemori K, Nokihara H, Miyake M, et al. Prognostic factors and clinical outcomes in patients with leptomeningeal metastasis from solid tumors. J Neurooncol, 2009;93:205-12.

7. Comte A, Jdid W, Guilhaume MN, Kriegel I, Piperno-Neumann S, Dieras $\mathrm{V}$, et al. Survival of breast cancer patients with meningeal carcinomatosis treated by intrathecal thiotepa. J Neurooncol. 2013;115:445-52.

8. De Azevedo CR, Cruz MR, Chinen LT, Peres SV, Peterlevitz MA, de Azevedo Pereira AE, et al. Meningeal carcinomatosis in breast cancer: prognostic factors and outcome. J Neurooncol. 2011;104:565-72.

9. Boogerd W, van den Bent MJ, Koehler PJ, Heimans JJ, van der Sande JJ, Aaronson NK, et al. The relevance of intraventricular chemotherapy for leptomeningeal metastasis in breast cancer: a randomised study. Eur J Cancer. 2004;40:2726-33.

10. Glantz MJ, Cole BF, Recht L, Akerley W, Mills P, Saris S, et al. High-dose intravenous methotrexate for patients with nonleukemic leptomeningeal cancer: is intrathecal chemotherapy necessary? J Clin Oncol, 1998;16:1561-7.

11. Lin N, Dunn IF, Glantz M, Allison DL, Jensen R, Johnson MD, et al. Benefit of ventriculoperitoneal cerebrospinal fluid shunting and intrathecal chemotherapy in neoplastic meningitis: a retrospective, case-controlled study. J Neurosurg. 2011;115:730-6.

12. Chamberlain MC. Leptomeningeal metastasis. Curr Opin Oncol. 2010;22:627-35.

13. Glantz MJ, Jaeckle KA, Chamberlain MC, Phuphanich S, Recht L, Swinnen LJ, et al. A randomized controlled trial comparing intrathecal sustained-release cytarabine (DepoCyt) to intrathecal methotrexate in patients with neoplastic meningitis from solid tumors. Clin Cancer Res. 1999;5:3394-402.

14. Fleischhack $G$, Jaehde $U$, Bode $U$. Pharmacokinetics following intraventricular administration of chemotherapy in patients with neoplastic meningitis. Clin Pharmacokinet. 2005;44:1-31.

15. Clarke JL. Leptomeningeal metastasis from systemic cancer. Continuum. 2012;18:328-42.

16. Pui $\mathrm{CH}$, Thiel $\mathrm{E}$. Central nervous system disease in hematologic malignancies: historical perspective and practical applications. Semin Oncol. 2009;36:S2-16.

17. Tallet A. Métastases cérébrales de cancer du sein. Cancer Radiother. 2013;17:708-14.

18. Gwak HS, Joo J, Kim S, Yoo H, Shin SH, Han JY, et al. Analysis of treatment outcomes of intraventricular chemotherapy in 105 patients for leptomeningeal carcinomatosis from non-small-cell lung cancer. J Thorac Oncol. 2013;8:599-605.

19. Gauthier H, Guilhaume MN, Bidard FC, Pierga JY, Girre V, Cottu PH, et al. Survival of breast cancer patients with meningeal carcinomatosis. Ann Oncol. 2010;21:2183-7.

20. Park KK, Yang SI, Seo KW, Kim YO, Yoon KY. A case of metastatic leptomeningeal carcinomatosis from early gastric carcinoma. World $\mathrm{J}$ Surg Oncol. 2012;10:74.

21. Leong L. Clinical relevance of targeting cancer metastases. Landes Bioscience. 2000

22. Clarke JL, Perez HR, Jacks LM, Panageas KS, Deangelis LM. Leptomeningeal metastases in the MRI era. Neurology. 2010;74:144954. 